\title{
Introduction: Toward a Definition of Biosemiosic Chance
}

\author{
Victoria N. Alexander
}

Received: 17 March 2014 / Accepted: 29 July 2014 / Published online: 27 August 2014

(C) Springer Science+Business Media Dordrecht 2014

\begin{abstract}
In this special issue, our objective is to clarify what biosemioticians may mean insofar as they claim that living systems are capable of making choices or that biosemiotic interpretations are partially indeterminate. A number of different senses of the term "chance" are discussed as we move toward a consensus. We find that biosemiosic chance may arise out of conditions involving quantum indeterminacy, randomness, deterministic chaos, or unpredictability, but biosemiosic chance is mainly due to the fact that living entities (i.e., cells or organisms) invest their environments with different meanings and values, which are not inherent in the material aspects. Accordingly, interpretive responses are in part self-determined. A self-determined interpretation may be thought of as a process in which localized biases constrain the probabilities of the outcomes of the interactions between an agent and its environment. The agent's internal formal constraints that are defined by similarity and proximity are relative and, as such, vague, and thus interpretation may be somewhat underdetermined and involve an element of chance.
\end{abstract}

Keywords Biosemiosis and interpretation - Interpretation as indeterminate - Semiotic constraints on probabilities $\cdot$ Chance affordances $\cdot$ Vagueness $\cdot$ Information selection

Biosemiotics depends upon the notion that interactions between living beings and their environments are not strictly determined by transformations of matter and energy - and thus are not fully describable by physical laws (Newtonian, quantum mechanical, or thermodynamic) alone. According to biosemiotics, living beings, organisms as well as single cells, tend to interact with matter as signs of potentially useful (or selfconfirming) processes. In a word, we argue that life can respond to its environment and does not only react. Responses are "purposeful" and "interpretive." Living entities invest the environment with different meanings and values, which are not inherent in the material aspects (see Wheeler this volume). Accordingly, interpretive responsesthe "choices" a living entity makes - are in part self-determined insofar as they occur as localized biases upon the probabilities of contingent outcomes and involve, therefore,

V. N. Alexander $(\bowtie)$

Dactyl Foundation, New York, NY, USA

e-mail: alexander@dactyl.org 
an element of chance (Alexander 2014). The ability of a living system to "choose" is determined in part by the particular and peculiar constraints (i.e., eigenbehaviors) it has developed over time. Although biosemiosic chance can be unpredictable, the type of "free" or "unlawful" behavior we're interested in here might be best described as "making your own luck."

The abilities of organisms to respond in useful ways exist to the extent that they do because they were selected for in the past; however, biosemioticians claim life is not simply programmed by natural selection, provided with set algorithmic ways of interfacing with the environment. If this were so, organisms would be mere robots, and unlike robots, organisms behave in unpredictable ways and can adapt. A living system's next state is not strictly determined by its internal state plus the state of its detectable environment. Life seems to be able to make use of (transform) noisy information in its environment, transcending any inherited algorithmic mode. How is this possible?

Part of the answer involves attributing the determination of interpretive acts to other types of causes than material and efficient (mechanical) causes, namely formal and final causes (Juarrero 1999; Alexander 2002, 2011; Thompson 2007; Deacon 2011), whose outcomes are not predictable in the sense that they involve biases developing through relations (e.g. physical similarity or proximity) and associations, which cannot be assigned a numerical value or transformed in lawful ways (e.g., $x$ 's degree of similarity to $y$ cannot be expressed in terms of an amount of energy). The phenomenon of formal self-organization can depend upon physically similar shapes being capable of more efficient bonding than others, or it can depend upon some physical barrier or other formal quality that only allows certain types of elements into the vicinity of a selforganizing system. Such biases can increase the chance of more regular physical arrangements forming out of otherwise random interactions. This regularity would not be strictly determined by the number of available kinds of elements as it is in thermodynamic processes, but would also be determined by the relative similarity and contiguity of elements as well. These kinds of formal relations can be imprecise, unpredictable, context dependent or historically contingent. If out of these formal biases a self-organized system emerges that is differentially and imperfectly self-replicating (and therefore capable of undergoing selection for function), as well as self-maintaining, then final cause also comes into play; meaning has emerged, and life has started to self-organize out of non-living matter.

Cell signaling is an example of a process that is determined by formal and final causes as well as efficient and material causes. A process may involve signaling if it has the following characteristics: It involves several steps, a chain of chemical and/ or physical interactions whose links are based upon formal constraints. Within the chain, any one of the links might be as arbitrary as those in a Rube Goldberg machine, as long as the goal state (e.g., homeostasis or survival of the cell) is ultimately achieved. The elements in each step are a means to an end, and what they are materially is not as important as what they stand for or are associated with, the ultimate positive feedback, which is the final cause. The probability of these interactions recurring is determined by the eventual function, the meaning the interactions have for the larger system, and thus we have a distinct kind of chance here affecting probabilities, biosemiosic chance, which depends upon formal and final causes. 
As Jesper Hoffmeyer notes, American semiotician C. S. Peirce believed that "chance and indeterminacy are the primordial condition of the world", and he thought, therefore, "the task is to explain how ordered structures emerge out of unordered, chaotic diversity" through habit formation (semiosis), and/or through what we would now call self-organization (Hoffmeyer 2009: 62-63).

Perhaps Peirce's intuitions have been partially confirmed by certain interpretations of quantum mechanics. If the primordial condition of matter is indeed indeterminate, then order does appear to emerge out of disorder. However, radical indeterminacy, such as we may find in the quantum mechanical world, is unlike the kind of "chance" that appears to be involved in self-organization - though it is, perhaps, equally surprising (see Auletta and Torcal this volume).

Peirce's "absolute chance" can be associated with the radical indeterminacy some have identified in the quantum world, which appears to be wholly irregular and uncorrelated, and thus allows radical indeterminacy to average out, resulting in probabilistic determinism. Semiosis tends to arise from correlations, which select contingencies, deepen biases, and result in novel (hence unpredictable) patterns. The latter does not necessarily follow from the former. As Kalevi Kull (2014) points out, contrary to what Peirce believed, the kinds of selections that result in biological "habits" are unlike the kinds of selections that result in physical laws.

Furthermore, complexity scientists argue that radically novel self-organization can emerge in fully deterministic systems. Thus, perhaps, it is neither true that a fundamental irregularity of indeterminacy (Firstness for Peirce) is a sufficient condition to explain the emergence of self-organized (unpredictable) order, nor is it, possibly, even a necessary one.

If the process of semiosis is what frees the living entity from a predictable material existence, then semiosis must, it would seem, produce the specific type of indeterminacy in interpretive responses: if it exists, biosemiosic chance must be emergent, coextensive with life, not already existing in the initial conditions of fundamental irregularity (Ostdiek this volume). We argue that inorganic matter and life are radically discontinuous. This is contrary to what Peirce argues, from a monist perspective that makes aliveness already existing (if only in potential) in initial indeterminacy or "spontaneity" of the physical realm.

We contend that the semiotic freedom of interpretations cannot depend on quantum fluctuations or deterministic chaos to indiscriminately free them. Instead discrimination must somehow flow from the living entity. Biosemiosic chance is not simply unpredictable. It is not simply random. It makes sense to the organism.

The term "interpretation" is used in biosemiotics to refer to a processes of habit formation, a process a meaningful (i.e., self-sustaining) selection, resulting in relative homeostasis in a changing environment. Interpretation is, at other times, a process of adaptation, learning and novelty generation. In the first instance, a new kind of regularity emerges that could not have been predicted by measuring the matter and energy of the initial conditions; in the second instance, a relative irregularity disrupts an established habit allowing a new regularity to emerge (Collier 2003). On the one hand, it is the process of self-organization that leads to the emergence of new constraints that narrow possibilities and make living actions more regular. On the other hand, it is the process of biosemiosic interpretation that repurposes constraints through associations: iconic or indexical (Alexander 2011: 78-88). 
We acknowledge that signs can and often do function algorithmically: when a sign has a physical connection to its (self-confirming) object, it is a rule-bound habit. When a sign does not have a physical connection, it is a sign by abduction, and a chance similarity or chance contiguity may afford life with novel ways of interacting with its world (Wheeler this volume).

The "chance" or spontaneity that Peirce called Firstness refers to irregularity or surprise, something having no relation to what comes before, but biosemiosic chance may be better described as a surprising regularity, the creation of a relation that did not exist before.

To some extent life's flexibility may depend upon some kinds of chance that exist in the primordial nature of all things, prior to the emergence of life. Several of the authors in this issue note that without some kind of radical irregularity at a fundamental level there would be no variety, and there would be no possibility for biases to form (see Auletta and Torcal and Coffman this volume). But with life, and only with life, are there signs, and signs bring their own kind of chance regularity into the mix, biosemiosic chance.

In their paper, "Chance and Events: The Way in which Nature Surprises Us", Gennaro Auletta and Lluc Torcal begin their analysis of chance as surprise by describing quantum mechanical events, which, because we can only observe their effects (on a photon detector) we know nothing directly of their causes. They contend that because all events involve countless factors and casual threads, all events are therefore in some sense like quantum events: they cannot be fully described by our general laws, since laws describe kinds of events and do not describe actual individual events. Thus, all individual events are contingent, or in Peirce's terms, they are "Firsts", not in a specific relation to something else. As Auletta and Torcal further point out, all heterogeneous phenomena emerge from symmetry breaking, and thus while chance may seem, retrospectively, to follow a rule, at the moment of selection, it would be a First, an event without prior relation.

Although in this issue we are making a distinction between the kind of chance event that occurs at the quantum level and the kind of chance event that biological systems take advantage of in their interpretations, as Auletta and Torcal note, at the moment of selection the processes are fundamentally similar. Biosemiosic chance is due to a building up of unpredictable biases, of habits over time, but the individual selection itself, viewed apart from other selections, may have this quality of Firstness identified by Auletta and Torcal.

In "Creating the Umwelt: From Chance to Choice" Stanley Salthe makes a distinction between the sort of trends (Peirce would say "habits") that build up over time in probabilistic ways, resulting in predictable outcomes - determinism, and the sort of habits that organic systems tend to develop.

Salthe differentiates between the externalist perspective, which deals with averages, and the internalist perspective, which deals with singularities. At the internalist level, habitual or teleological responses are never quite the same twice but are continually being revised. According to Salthe, this perspective is a peripheral awareness, unfocused and vague. He notes that it is not possible for external observers at contiguous levels to precisely predict the behavior of the lower levels. The "choices" of the lower level "appear as chance" to the upper level because the semiotic rules informing the behavior are not known to the external observer. In biological systems, these rules 
would be based on some standards that are particular to the system itself, based on similarity, contiguity, and/or arbitrary associations with some goal state.

Salthe stresses that it is generally this loss of information between levels that creates unpredictability and not fluctuations from lower levels, since their influence on higher levels is prohibited by the difference in scale. As others in this volume point out, however, this does not mean that quantum events never affect the macro world. The results of single-point genetic mutations or minute disruptions of the brain's meta-stable state can be selected as meaningful (or harmful) by an organic system, but biological constraints are required to do the selecting, otherwise as rare and irregular, these fluctuations would be averaged out and would be inconsequential.

Further elaborating on the view that biosemiosic chance arises from underdetermined and/or contingent selections made from vague sense of the surround, in "The Manufacture of Chance" Gerald Ostdiek argues, contra Peirce, that "genuine Firstness", real spontaneity, seems to appear only as living things manufacture it via semiotic action. According to Ostdiek's view, radical indeterminacy, as discussed by Auletta and Torcal, can be considered "degenerate Firstness." Ostdiek critiques Peircean pansemiosis, which fails to make distinctions between the kind of Firstness that results in predictable determinism and the kind of Firstness, biosemiosic chance, that results in and from purposeful behavior. It is life, not physics, that is the source of true spontaneity, argues Ostdiek, because the behavior of seeking out and incorporating matter is radically discontinuous from the actions of non-living matter.

In "On the Meaning of Chance in Biology" James Coffman notes that chance is often defined, as per Auletta and Torcal and Salthe, as an event that cannot be described by known laws or predicted from them, so chance is defined negatively in terms or our general laws and experience. Coffman observes that throughout history, from time to time, scientists have asserted that some phenomenon is impossible or has no chance of occurring, only to discover later, after science has added new ways to contextualize knowledge, that the "impossible" is actually quite probable. New contexts are constantly emerging, but this is especially true with the advent of life.

Coffman looks back at Monod who was among the first to point out that genetic information is arbitrary with respect to the laws of physics and chemistry, and therefore life's self-sustaining behavior is not predictable from those laws. As a creator of new contexts and immeasurable complexity, life creates new chance through its interpretative actions in the world.

Finally, in "A Connoisseur of Magical Coincidences" Wendy Wheeler continues and extends such thoughts on biological context creation considering the special poetic abilities of humans. She discusses how meaning-making is a continual processes that changes the past, changes our meaning of the past, and creates possibilities for the future, which did not formally exist before the new linkage, based on some similarity (simile, metaphor) or contiguity (metonymy, synecdoche), was made. Further, she claims that the creative use of chance, exemplified by a poem by Thomas Hardy, "makes all evolutionary life, biological and cultural possible." According to Wheeler, poetic activities precede the emergence of human poets in the world. Biology is no less poetic in dealing with the chance affordances it finds, and we can understand biological processes better by studying how humans create new meanings with language.

Although these papers enter the conversation from very different points, they share the idea that there are several kinds of chance, ontological indeterminacy, randomness, 
deterministic chaos, whose common characteristic is unpredictability. This type of chance is highly irregular and can averaged out resulting in predictable behavior over time. The authors agree that a different kind of chance arises in contextual determinations, when meaning-making interpretive acts play a role in bringing something wholly new into existence.

\section{References}

Alexander, V. (2002). Narrative telos: the ordering tendencies of chance. PhD diss. The Graduate Center, City University New York.

Alexander, V. (2011). The biologist's mistress: rethinking self-organization in art, literature, and nature. Litchfield Park: Emergent Publications.

Alexander, V (2014). The science of making choices. Based on presentation, Toward a biosemiotic definition of chance, at 2013 Biosemiotics Gathering in Castiglioncello, Italy. Available at https://www.youtube. $\mathrm{com} /$ watch? $\mathrm{v}=\mathrm{dx}$ AIO_SLqsI

Collier, J. (2003). Fundamental properties of self-organization. In V. Arshinov \& C. Fuchs (Eds.), Causality, emergence, self-organisation (pp. 150-166). Moscow: NIA-Priroda.

Deacon, T. (2011). Incomplete nature: how mind emerged from matter. New York: W. W. Norton \& Company.

Hoffmeyer, J. (2009). Biosemiotics: an examination into the signs of life and the life of signs. Scranton: University of Scranton Press.

Juarrero, A. (1999). Dynamics in action: intentional behavior as a complex system. Cambridge: MIT Press.

Kull, K. (2014). Physical laws are not habits, while rules of life are. In T. Thellefsen \& B. Sorensen (Eds.), Charles sanders peirce in his own words: 100 years of semiotics, communication and cognition (pp. 8794). Berlin: Walter de Gruyter.

Thompson, E. (2007). Mind in life: biology, phenomenology, and the sciences of mind. Cambridge: Harvard University Press. 